\title{
INCREASING ENTROPY: HEAT DEATH OR PERPETUAL HARMONIES?
}

\author{
R.E. ULANOWICZ ${ }^{1,2}$ \\ ${ }^{1}$ Department of Botany and Zoology, University of Florida, Gainesville, USA. \\ ${ }^{2}$ University of Maryland Center for Environmental Science, Solomons, USA.
}

\begin{abstract}
Classically, the increase of entropy implies an ineluctable dissipation of energy and materials into what is known as 'heat death'. A strictly logical take on the Boltzmann entropy reveals, however, that the measure amalgamates order with disorganization. Hence, under some nonequilibrium circumstances, the production of order becomes an inevitable feature of increasing entropy. In particular, perpetual harmonies can emerge from the collapse of nonequilibrium configurations. Data from networks of trophic interactions in real ecosystems reveal a preferred balance between dissipation and order at an approximate ratio of $1: \mathrm{e}-$ a phenomenon that possibly could inform the search for sustainable systems.

Keywords: autocatalysis, complexity, dissipation, dualism, ecosystems, entropy, heat death, indeterminacy, information, monism, nonequilibrium, second law, sustainability, thermodynamics.
\end{abstract}

In memory of James J. Kay, who long ago beckoned the author down this path.

'If someone points out to you that your pet theory of the universe is in disagreement with Maxwell's equations - then so much the worse for Maxwell's equations. If it is found to be contradicted by observation, well, these experimentalists do bungle things sometimes. But if your theory is found to be against the second law of thermodynamics I can give you no hope; there is nothing for it but to collapse in deepest humiliation'.

Arthur S. Eddington [1]

\section{THE CONTEMPORARY WORLDVIEW}

Most accounts of the history of science connect the demise of the Newtonian worldview to the advent of relativity and quantum theories near the turn of the 20th century. But thermodynamicists are more inclined to reckon the departure as beginning some eight decades earlier, when Sadie Carnot [2] described behaviors that did not belong in the conservative theatre that reversible Newtonian mechanics had erected. Contrary to Newton, every real change brings with it an inevitable loss.

Carnot's experiments with simple physical systems later gave rise to mathematical constructs by Boltzmann [3] and Gibbs [4] that depicted a universe where loss was ubiquitous and ineluctable. Everything was winding down, it appeared. A simple system, isolated from the external world inevitably takes on the most disordered configuration possible. Everything ultimately degenerates into heat, which in its turn disperses into an ever-expanding universe. This final condition was termed 'heat death'.

This scenario fit well with the existentialist philosophies that arose out of the Great War at the beginning of the 20th century: Reality marches uniformly into disorder and its accompanying meaninglessness. That view, still fashionable among so many academics, has been dubbed the "cosmology of despair' by John Haught [5].

Of course, not everyone fell into step with this new cosmology. Many pointed instead to the phenomenon of life and to how order arises almost spontaneously among ontogenetic processes. For example, Erwin Schroedinger [6], in his essay 'What is Life?', pointed to the appearance of what he 
called (somewhat infelicitously in this writer's opinion) 'negentropy'. Thereafter, the challenge became how to explain the appearance of negentropy or order within the framework of the overarching second law of thermodynamics.

Nicolis and Prigogine [7] were able to reconcile negentropy with the second law by drawing sharp distinction between the internal state of a self-organizing system and that of the remainder of the universe. It is eminently possible, Prigogine maintained, for entropy to decrease within a living system so long as that system exports more than proportionate entropy to its surroundings. That is, order may increase locally so long as the order of the larger whole of which it is part suffers a net decrease.

Bookkeeping having thus been settled, attention then turned to describing what gives rise to local order. Stuart Kauffman [8] posited the need for a 'fourth law of thermodynamics' to describe developing living systems. Indeed, a host of thermodynamic-like propositions have been put forth: Jørgensen and Mejer [9] proposed a centripetal notion to the effect that living systems accumulate and store as much exergy (potential for work) as possible. Eric Chaisson [10] suggested that development always proceeds so as to augment 'energy rate densities'. Ulanowicz [11] described development as an increase in 'ascendency' - an information-theoretic analog to the Gibbs work function. Fath et al. [12] have made a comparison of the various attempts and concluded that they all deal directly or indirectly with the distance from equilibrium.

A common thread of the principles just mentioned is that they work independently of, and in opposition to, the second law. Others, however, have maintained that the formation of order results in subservience to the second law. Salthe [13] sees the second law as the ultimate final cause behind all that happens in the universe. For Swenson [14] the sole purpose of living systems is to accelerate the production of entropy. Likewise, Schneider and Sagan [15] perceive the increase in entropy as the major direction behind evolutionary change. Schneider and Kay [16] described the drive behind the development of living systems as a unitary statement of the two laws of thermodynamics whereby exergetic gradients are degraded as quickly as possible whenever and wherever they might appear. Common to all these propositions is the notion that the second law is the fundamental essence of universal dynamics, while apparent increases in order are accidental and subservient to the drive of the second law per se.

The proposition here differs in a subtle but fundamental way from these latter suggestions, namely, as first suggested to the writer by James Kay, the prevailing view of the second law is an oversimplified version of its true nature. Simply put, entropy is not entirely about disorder. Away from equilibrium, there is an obverse and largely unappreciated side to the second law that, in certain circumstances, mandates the creation of order. Chaisson [10] remarked upon this 'duality' in the second law, and Ulanowicz [17] earlier attempted to formulate the obverse as, 'In any real process, it is impossible to dissipate a set amount of energy in finite time without creating any structures in the process.' Morowitz [18] pointed out the propensity of energy dissipation to engender cycles. The task here is to provide further theoretical development to this notion and to examine the ensuing results in the light of ecological data.

\section{THE NATURE OF ENTROPY}

Before turning attention to theory, it should be noted that the field of thermodynamics is primarily an empirical domain. As became evident in the wake of Carnot, in any conflict between theory and phenomenological observation, advantage always accrues to the phenomenological. Thus, Carnot's documentation of irreversible physical phenomena put the reversible (and conservative) Newtonian theory (and with it the burgeoning atomic theory) at risk. Redeeming these theories became the paramount challenge to the physicists of the 19th century over the next five or six decades. The challenge culminated in the 'reconciliation' of Newtonian law and thermodynamics in the statistical mechanics 
of Boltzmann and Gibbs. Oliver Penrose [19] notes, however, that this 'synthesis' is falling under increasing scrutiny.

Although over the next two sections attention will focus on theoretical issues, it should be recognized that only additional empiricism is able to modify earlier phenomenology. Empirical observations favoring the dual nature of entropy will be provided in this paper. Suffice it for now to note that the phenomenological definition of entropy $(d S=d Q / T)$ is cast in such a way as to reveal only one side of the purported duality. The classical definition is valid only at equilibrium, which precludes its application to nonequilibrium situations, where the more extensive nature of Eddington's beloved principle becomes evident.

The most propitious starting point for recognizing the dual nature of entropy is the BoltzmannGibbs formulation $[3,20]$, with which the formulators intended to reconcile thermodynamics with Newtonian law. The form most convenient to the present discussion is

$$
H=k \sum_{i} p_{i}\left(-\log \left[p_{i}\right]\right)
$$

where $H$ is the statistical representation of entropy, $p_{i}$ is the probability of event $i$, which is properly normalized to fall between zero and one, and $k$ is a scalar constant. The formula has been grouped in this particular fashion to emphasize the fact that the statistical entropy is the average value of the term in parenthesis,

$$
s_{i}=-\log \left[p_{i}\right],
$$

where $s_{i}$ has been called the 'surprisal' of possible outcome $i$ by Tribus [21]. By this reckoning, any event with small probability of happening, $p_{i}$, will yield a large value for $s_{i}$ (One will be very surprised when event $i$ occurs.) Conversely, a probability of $i$ near unity (certainty) will result in a small value of $s_{i}$; that is, one is hardly surprised that event $i$ almost always occurs.

In assessing the meaning of the surprisal, it becomes all too easy to regard the negative sign as a mathematical convenience that assures that $s_{i}$ will always remain positive for any legitimate probability. It is too easily dismissed as a device. But, one may instead examine eqn (2) for its logical implication: For example, one can regard $p_{i}$ as quantifying the strength of event $i$ 's possible existence. Because the logarithm is a monotonic function of its argument, it follows that $s_{i}$ then becomes a measure of the event $i$ 's nonexistence. That is, if $s_{i}$ is very large, event $i$ does not occur most of the time.

The significance of this interpretation cannot be overstated. In a sense, it is analogous with the invention of zero by the Arabs. This is because conventional physics deals with what is. It is overwhelmingly positivistic. As Gregory Bateson [22] has noted, statements concerning negation are quite rare in physics, for example, Pauli's exclusion principle, Heisenberg's uncertainty, and so on. Therefore, physics is ill equipped to describe that which is missing. Yet the absence of some object or process can be of critical importance in so many biological situations. In fact, the notion of 'pattern' often rests upon the locations of missing elements. For example, whether a predator is present or absent is often of vital importance to the persistence of a given species. Spatial patterns usually consist of juxtapositions of presences and absences, etc. Those who seek to reduce ecology (or even more generally, biology) to physics will inevitably be frustrated by the inadequacy of the latter to address patterns in nature. For that reason, Ulanowicz et al. [23] have characterized the rejection of information theory by ecologists as 'one-eyed ecology'.

It is crucial to the nature of entropy that the measure for the nonexistence of $i$ does not appear in isolation in the Boltzmann-Gibbs formula. Rather, each possibility of the nonexistence of $i$ is paired 
with and modulated by the measure of its existence, $p_{i}$. When the yardstick for the presence of an event is multiplied by the corresponding measure of its nonexistence, the result

$$
h_{i}=-k p_{i} \log \left(p_{i}\right)
$$

becomes, de facto, a gauge of that event's indeterminacy. To see this, one notes that when $p_{i} \approx 1$, the event is almost certain, and $h_{i} \approx 0$; when $p_{i} \approx 0$, the event is almost surely absent, and again $h_{i} \approx 0$. It is only for intermediate, less determinate values of $p_{i}$ that $h_{i}$ becomes appreciable, achieving its maximum value at $p_{i}=(1 / \mathrm{e})$.

It should not, therefore, be too surprising that eqn (3) is germane to change and evolution. For, when $p_{i} \approx 1$, the event in question is a virtual constant in its context and unlikely to change $\left(h_{i} \approx 0\right)$. Conversely, whenever $p_{i} \approx 0$, the event possesses great potential to change matters $\left(s_{i} \gg 1\right)$, but it hardly ever appears as a player in the system dynamics, so that, again, $h_{i} \approx 0$. It is only when $p_{i}$ is intermediate that the event is both present frequently enough and is endowed with sufficient potential for change. Thus, $h_{i}$ gauges the capacity for event $i$ to be a significant player in system change.

As most readers are probably aware, Shannon [24] later recapitulated eqn (1) as the beginning of modern information theory. In epistemic terms, the focus of $H$ is on the observer's average uncertainty about events in a given probability distribution, but for reasons just discussed, emphasis can be directed instead towards the intrinsic capacity of the system itself for evolution.

Boltzmann and Gibbs applied their formula to the distributions of atoms in an ideal gas, that is, a gas consisting of point-sized atoms having no interactions with one another. The only endpoint possible under these assumptions was the maximal dispersion of the gas - what came to be termed 'heat death'. Physicists, with their predilection for theory over phenomenology, have taken the BoltzmannGibbs formula as the very definition of entropy and with it came the baggage that $H$ represents only the disorder inherent in a system.

The foregoing interpretation of the Boltzmann-Gibbs formula reveals, however, that entropy is inherently dualistic. It contains within itself the antipodes of being and nonbeing, and so the question naturally arises, 'Do there exist circumstances under which changes in the former state might dominate over variations in the latter?'

Before further unpacking the formal definition of entropy, one would be justified in asking why not simply choose $(1-p)$ instead of $[-\log (p)]$ as the most appropriate measure of nonexistence? The answer is that the resultant product with $p$ (that is $\left[p-p^{2}\right]$ ) is perfectly symmetrical around the value $p=0.5$. Calculations pursuant to such a symmetric combination would be capable of describing only a reversible universe. Boltzmann and Gibbs, however, were seeking to quantify an irreversible universe. By choosing the univariate convex logarithmic function, Boltzmann thereby imparted a bias to nonbeing over being. One notices, for example, that $\max [-x \log \{x\}]=\{1 / \mathrm{e}\} \approx 0.37$, so that the measure of indeterminacy is skewed towards lower values of $p_{i}$.

\section{QUANTIFYING ORGANIZATION}

If, as suggested above, order is part and parcel of the statistical entropy, how can one quantify its degree? Here it is crucial to note that if the Boltzmann-Gibbs formula is applied to a system whose elements cannot interact (as with an ideal gas), the production of order within increasing entropy can never emerge. It is essential that the events in question have a capability to interact with each other before it becomes possible to distinguish any order immanent in $H$ from its complement of disorder, which conventionally has been considered the sole aspect of entropy.

For example, if $p\left(a_{i}\right)$ is the probability that $a_{i}$ occurs, and $p\left(a_{i} \mid b_{j}\right)$ is the conditional probability that $a_{i}$ occurs whenever $b_{j}$ has occurred, then the condition that $p\left(a_{i} \mid b_{j}\right) \neq p\left(a_{i}\right)$ implies that some reciprocal 
influence exists between $a$ and $b$. Extant such interaction, one can then decompose $H$ into two complementary terms, using the well-known result from information theory,

$$
\begin{aligned}
H & =-k \sum_{i} p\left(a_{i}\right) \log \left[p\left(a_{i}\right)\right] \\
& =k \sum_{i} \sum_{j} p\left(a_{i}, b_{j}\right) \log \left[\frac{p\left(a_{i} \mid b_{j}\right)}{p\left(b_{j}\right)}\right]-k \sum_{i} \sum_{j} p\left(a_{i}, b_{j}\right) \log \left[\frac{p\left(a_{i}\right) p\left(a_{i} \mid b_{j}\right)}{p\left(b_{j}\right)}\right],
\end{aligned}
$$

where $p\left(a_{i}, b_{j}\right)=p\left(b_{j}\right) p\left(a_{i} \mid b_{j}\right)$ is the joint probability that $a_{i}$ and $b_{j}$ co-occur.

Both terms in the decomposition, like their sum, $H$, are intrinsically non-negative. The first term in the sum is called the mutual information between $a_{i}$ and $b_{i}$. It is a logarithmic analog of the correlation between events $a_{i}$ and $b_{j}$. It quantifies the extent to which $a_{i}$ is constrained by, or ordered by, $b_{j}$. Conversely, the second term, or conditional entropy, quantifies the extent to which $a_{i}$ is independent of or indeterminate by $b_{j}$.

This decomposition has two important consequences; it portrays how the order inherent in $H$ can become explicit via comparison with yet another distribution. In doing so, it reveals how any measure of order and/or disorder inhering in a distribution is always relative to some other reference distribution (echoes of the 'third law' of thermodynamics.)

Boltzmann's calculus was constructed to treat occurrences of objects (atoms or molecules) in a positivist way as befits equilibrium thermodynamics. Under equilibrium thermodynamics, processes, or transitions between states, play a distinctly secondary role. The proposition tendered here is that the preoccupation under conventional thermodynamics with objects or states over and above transitions or processes necessarily blinds one to the genesis of order in the natural world. Henceforth, focus turns toward the much neglected process variables.

Consonant with the shift in focus, it becomes necessary to adapt the formulae (1)-(4) to processes. Fortunately, this is a rather straightforward exercise [25]. The first step is to notice that the probability of the transition $a_{i} \rightarrow b_{j}$ can readily be represented by the joint probability, $p\left(a_{i} b_{j}\right)$, so that the ensuing statistical entropy of a collection of processes becomes

$$
H^{\prime}=-k \sum_{i, j} p\left(a_{i}, b_{j}\right) \log \left[p\left(a_{i}, b_{j}\right)\right] .
$$

Whereupon the corresponding mutual information, $I$, becomes

$$
I=k \sum_{i, j} p\left(a_{i}, b_{j}\right) \log \left[\frac{p\left(a_{i}, b_{j}\right)}{p\left(a_{i}\right) p\left(b_{j}\right)}\right],
$$

and the conditional entropy is written as

$$
\Phi=-k \sum_{i, j} p\left(a_{i}, b_{j}\right) \log \left[\frac{p\left(a_{i}, b_{j}\right)^{2}}{p\left(a_{i}\right) p\left(b_{j}\right)}\right] .
$$

One notes that each of the three definitions, eqns (5)-(7), is symmetrical in $a_{i}$ and $b_{j}$.

In order to assign measurable values to these indices, it is necessary to first estimate the various probabilities in terms of observable frequencies. As a preliminary, the observed magnitude of the 
process $a_{i} \rightarrow b_{j}$ will be denoted by $T_{i j}$. For example, $T_{i j}$ might be the measured rate by which reactant $i$ transforms into product $j$, or the rate at which prey $i$ is eaten by predator $j$. The marginal sums of these transitions will be indicated by a dot in place of the index that is summed. Whence, $T_{. j}=\Sigma_{i} T_{i j}$ represents the total amount of every category that becomes $j$ in a given unit of time. The total activity of all processes (what Finn [26] and others have called the 'total system throughput') becomes (T..), and the frequency of the transition from $a_{i} \rightarrow b_{j}$ [the joint probability $\left.p\left(a_{i}, b_{j}\right)\right]$ is reckoned as $\left(T_{i j} / T_{\text {... }}\right)$. Using this nomenclature, the decomposition

$$
H^{\prime}=I+\Phi
$$

takes the form

$$
-k \sum_{i, j}\left(\frac{T_{i j}}{T_{. .}}\right) \log \left(\frac{T_{i j}}{T_{. .}}\right)=k \sum_{i, j}\left(\frac{T_{i j}}{T_{. .}}\right) \log \left[\frac{T_{i j} T_{. .}}{T_{i .} T_{. j}}\right]-k \sum_{i, j}\left(\frac{T_{i j}}{T_{. .}}\right) \log \left[\frac{T_{i j}^{2}}{T_{i . .} T_{. j}}\right] .
$$

Given any collection of transitions, $T_{i j}$, it thus becomes possible to partition the entropy of the array $\left(H^{\prime}\right)$ into an amount that expresses the coherency (order) among the processes $(I)$ as distinguished from how much remains as residual indeterminacy $(\Phi)$.

\section{THE ORIGINS OF ORDER}

Reasoning from dimensional consistency, any theory that is delimited to static, unchanging states will remain incapable of elucidating the process that is increasing order. It follows that processes must be rehabilitated as part of, or even the focus of, an expanded thermodynamics. Whereas the intent behind 'the thermodynamics of irreversible processes' has been to expand thermodynamics to encompass real processes, the enterprise has been handicapped by a focus that remained on changes of states, rather than concentrating on the processes themselves.

Apropos the balance between states and processes, Bernardus Tellegen has demonstrated that it may be more equivocal than usually has been assumed [27]. In his treatment of network thermodynamics, Tellegen demonstrated how strict linearity between states and processes would make it possible to describe either one in terms of the other in a fully equivalent fashion. That is, under linearity, one is as free to describe states in terms of processes as to follow the usual convention by describing processes in terms of states. For example, one usually regards electrical current as flowing because of a difference in potential between two points, but it makes fully as much sense to regard the potential as the convergence (or divergence) of currents at a point.

Of course, the dynamics of nature are rarely only linear, but the point of Tellegen's exercise is that it is possible to formulate an alternative or dual description of nature in terms of processes, rather than states. Actually, there is nothing particularly new in such a reversal. Heraclitus, leader of the Milesian School of Greek thought, posited change as the fundamental verity of nature. The Milesians antedated the Eleatic community (associated with Plato) that sought to interpret nature in terms of unchanging essences. Science, over its past 300 years, has been predominately Eleatic in perspective, and those who have advocated more process-based descriptions of reality, such as Peirce [28] and Whitehead [29], have largely been ignored.

A truly irreversible thermodynamics should focus, however, upon the irreversible, i.e. stress processes over and above states. Although recognizing that process and form are both necessary for full description, it is worthwhile to explore the possibility that a useful explanation of processes can be sought primarily in terms of other processes. That is, instead of searching for agency among objects or states, Popper [30] has suggested that one look instead to other processes or combinations thereof. 
Along these lines, it should be noted that the notion of processes affecting and begetting other processes has a very legitimate history in thermodynamics - witness the Kolmogorov [31] decay of large eddies into smaller ones or Onsager's [32] coupling of processes.

That processes can affect other processes hints at feedback, and Kauffman [33] and Ulanowicz [34], among others, have pointed to a particular form of feedback, autocatalysis, as the very generatrix of order. Autocatalysis is that subset of all positive feedbacks wherein each process exerts a positive influence upon its downstream neighbor in the cycle of processes. For example, the three hypothetical processes depicted in Fig. 1 are configured in autocatalytic fashion. It should be stressed that it is not necessary for the effect of any participant upon its next neighbor be strictly mechanical. There need only be a probabilistic propensity (sensu Popper [30]) that the activity of any member augment the following process. In this example, the action of process A has a propensity to augment a second process $\mathrm{B}$. B in its turn tends to accelerate $\mathrm{C}$ in similar fashion, and $\mathrm{C}$ has the same effect upon A. Kauffman [33] discusses how, in any random assembly of a number of processes, the probability of encountering such autocatalytic configurations increases precipitously as the number of processes grows.

To appreciate how autocatalysis creates further order, it is necessary to be aware of several intrinsic features of autocatalytic feedback [17]. In particular, autocatalysis is capable of exerting selection pressure upon its own ever-changing constituents. To see this, one considers that some small change occurs spontaneously in process B in Fig. 1. If that change makes B either more sensitive to A or a more effective catalyst of $\mathrm{C}$, then the change will receive enhanced stimulus from $\mathrm{A}$. Conversely, if the change in B makes it either less sensitive to the effects of A or a weaker catalyst of C, then that change will likely receive diminished support from A.

Whenever resources are not limiting, autocatalysis is, by definition, growth-enhancing. Such enhancement can interact with selection to create a distinct centripetality or vortex of materials and resources being drawn into the autocatalytic cycle. To see this, one notes how any enhancement in the activity of B (Fig. 1) is likely to be coupled with an increase in the amounts of material and energy that flow to sustain B. That is, the nature of autocatalytic configuration is to reward and support those changes that bring ever more resources into B. As the same circumstance pertains as well to all the other members of the feedback loop, the autocatalytic cycle becomes the center of a centripetal vortex that pulls as many resources as possible into its domain (Fig. 2).

Although growth is the prevailing response to abundant resources, dynamics are somewhat different when external resources are saturated or diminishing. Under such circumstances, centripetality acts instead to reroute flows in a system away from nonparticipating exchanges into those links that engage in the autocatalytic configuration. The dynamics under declining resources roughly follow the progression depicted in Fig. 3.

In Fig. 3(b) flows such as the former one from $3 \rightarrow 2$ [Fig. 3(a)] have been diverted to augment adjacent flows that participate more in autocatalysis, such as the one from $3 \rightarrow 4$. One can demonstrate that, for any given number of flows, a theoretical upper limit to $I$ occurs when all flows comprise

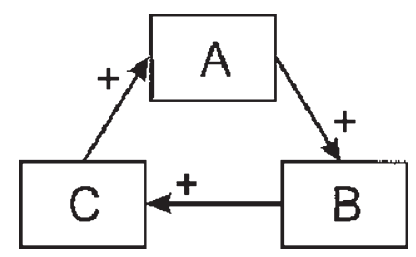

Figure 1: Three hypothetical processes in autocatalytic configuration. 


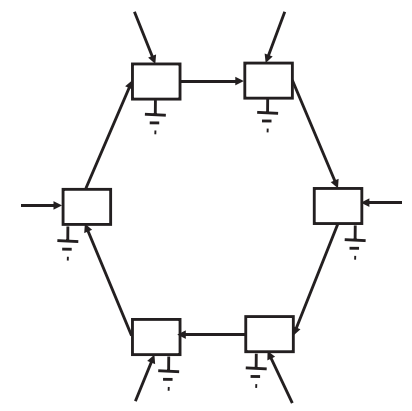

Figure 2: An autocatalytic configuration of processes induces the attraction of material and resources into its circuit - a property termed 'centripetality'.

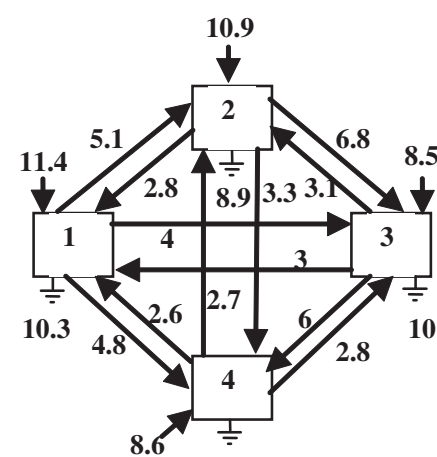

10.2

I $=0.404 ; \alpha=0.098$

(a)
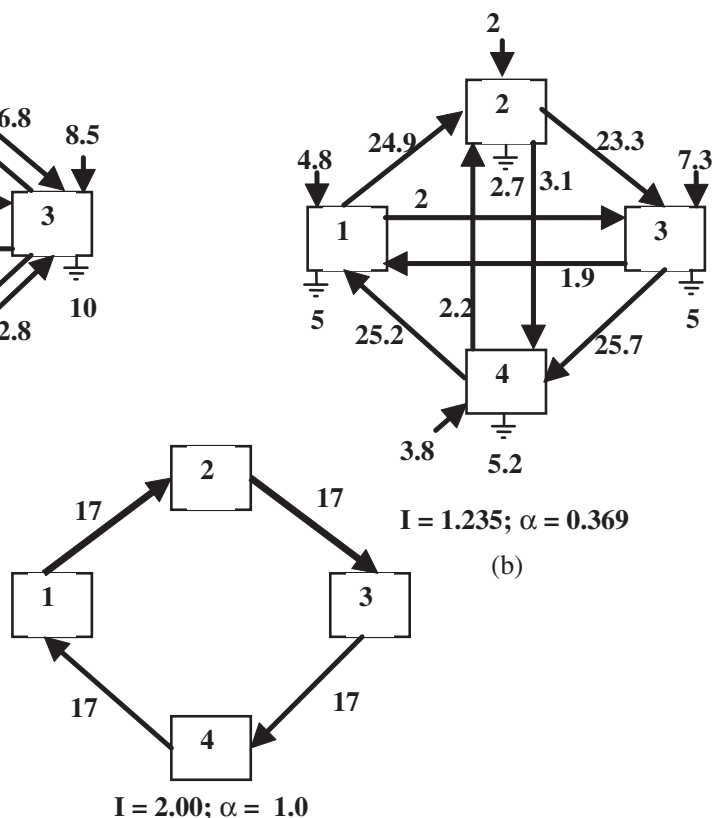

I = 1.235; $\alpha=0.369$

(b)

(c)

Figure 3: The progression of a hypothetical simple system under declining availability of external resources: (a) a very undeveloped configuration, (b) flows are being rerouted along pathways that participate more in autocatalysis and (c) the ideal, hypothetical limit of a simplified, equilibrium, equiponderant cycle of flow with no external connections (a perpetual harmony). ' $\alpha$ ' is the degree of order, as defined in the text. 
closed cycles of equiponderant transfers. In that limit, there are no longer any transfers with the outside world, so that, by definition, the configuration represents a state of equilibrium. With increasing order, open dissipative systems approach the limit of becoming closed equilibrium configurations.

A measure of especial significance is the fraction of the total flow diversity $\left(H^{\prime}\right)$ that is constrained in ordered fashion $(I)$, i.e. the ratio, $\alpha=I / H^{\prime}$. One may call this ratio the 'degree of order', and it ranges from 0 to 1 . The net effect of autocatalytic action (the internal creation of ordered constraints) is to increase $\alpha$. The upper theoretical limit to this drive [Fig. 3(c)] is $\alpha=1$ (a state of closed equilibrium). Conversely, the tendency of a system toward the limit $\alpha \rightarrow 0$ represents the approach to 'heat death'.

In simple physical systems that have little or no interaction between elements, the inevitable endpoint of continued entropy production is $\alpha=0$. The production of entropy in more complicated systems that exhibit significant interaction among their elements is, however, more complex. In such situations, one perceives dual, antagonistic directions. On one hand is the familiar slide into total dissolution $(\alpha \rightarrow 0)$, but as Ulanowicz [17] has argued, the presence of any autocatalytic cycles would tend to drive the system in exactly the opposite direction - towards $\alpha \rightarrow 1$.

It is important to note that both extrema of $\alpha$ are conditions of equilibrium. The first $(\alpha=0)$ is pure dissolution, whereas the second $(\alpha=1)$ represents a 'perpetual harmony'. Systems approaching $\alpha=0$ are unlikely to possess sufficient cohesion to maintain their integrities over time. In contrast, systems too near $\alpha=1$ are so fully constrained that they no longer can evolve. Systems capable of evolution over time will occupy the realm between 0 and 1 , but should not approach too near either endpoint.

It remains to quantify the evolutionary potential of real, irreversible systems. If $\alpha$ represents the degree of organizational constraint within a system, then its complement $(1-\alpha)$ should be the appropriate measure of the system's disorganization or freedom to change. At least, such would be the measure in a reversible, conservative world. But, as Boltzmann demonstrated, that may not be the most propitious index to employ in the irreversible realm. In a second law universe, where nature gives bias to indeterminacy, $-\log (\alpha)$ should be a more appropriate way to characterize disorganization. One notes that $-\log (1)=0$, indicating that such a measure of disorganization also vanishes in the limit of perfect harmony.

Those irreversible systems that are robust and most likely to persist over time possess a modicum of constraint $(\alpha)$ in combination with sufficient freedom to change $[-\log (\alpha)]$. In analogy with statistical mechanics, such robustness can be defined as the product of these countervailing attributes, or

$$
R=-\alpha \log (\alpha) .
$$

In this scheme, systems that lack internal cohesion $(\alpha \approx 0, R \approx 0)$ cannot be robust. Neither can those that are unable to change in response to unanticipated perturbation $(\alpha \approx 1, \underline{R} \approx 0)$. According to definition (9), those most likely to persist (be sustainable) would be the ones near maximal $R$, when $\alpha=(1 / \mathrm{e})$. It remains to be seen how actual ecosystems are distributed over the ranges of $\alpha$ and $R$.

\section{ORDER IN REAL ECOSYSTEMS}

Before examining data on real living systems, it is of interest to pause and consider how $\alpha$ varies among randomly constructed, weighted networks. As might be expected, networks for which the magnitudes of the $n^{2}$ flows are generated by the uniform random distribution all cluster near $\alpha \approx 0$ (and $R \approx 0$ ), more tightly so as $n$ grows larger.

As a sample of data on real ecosystems, Zorach and Ulanowicz [35] collected weighted networks of trophic exchanges in 48 ecological communities. The calculated values of $\alpha$ and $R$ for these systems are plotted against each other in Fig. 4. (That the points coincide precisely on the curve is merely a matter of algebraic identity. The plot is intended to show how the systems are distributed with respect to the maximum of the curve.) Most, in fact, do cluster around $\alpha=1 / \mathrm{e}$, with a few scattered 


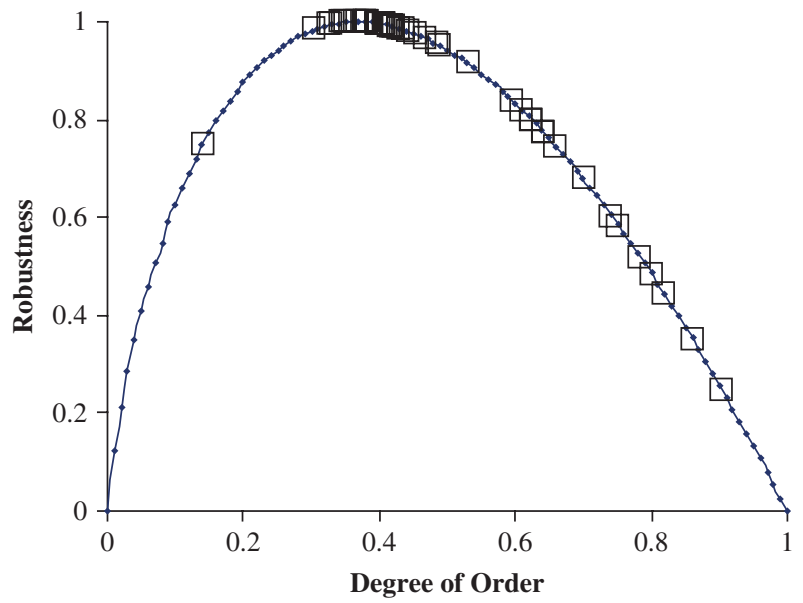

Figure 4: The degrees of order and their corresponding magnitudes of robustness for the 48 sample weighted ecosystem flow networks used by Zorach and Ulanowicz [35].

towards higher values of the degree of order. Robert Christian (personal communication) pointed out to the author that all the systems with higher values of degree of order were communities that had been represented using very few constituent elements $(n=4-8)$. Such depictions are likely to be lacking in richness and detail.

For those systems described in greater detail, the values of degree of order and robustness tended to cluster more tightly near maximal robustness. Figure 5 shows the distribution of the 17 systems from the collection for which $n>12$. The minimal value of the robustness for this subset was $R=$ 0.954 . The tendency of actual systems to bunch so tightly had been noted by Zorach and Ulanowicz [35] and by Ulanowicz [36], who called the region of clustering a 'window of viability'.

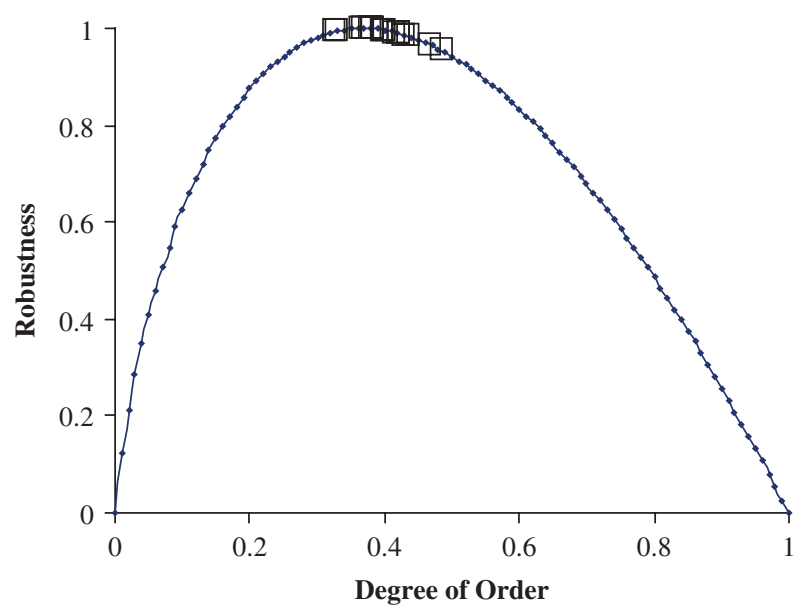

Figure 5: The degrees of order and corresponding magnitudes of robustness for the subset of 17 ecosystem flow networks in Fig. 4 that each possess $>12$ compartments. 


\section{THE DIALOG OF NATURE}

The ramifications of the foregoing analysis are profound - perhaps none more so than the inference that the universe is dualistic, rather than monistic in its dynamics. The Western narrative on nature has followed predominately monistic scripts - entropy is maximized; efficiency is maximized; the Hamiltonian of a trajectory is maximized; costs are minimized, etc. With the application of Occam's Razor to simplify natural dynamics, dualisms have been rejected as unnecessarily complicated. But if the encounter of science with complex systems has indicated anything, it is that Occam's Razor is a double-edged blade [37]. Clinging to monism is likely to prove a counterproductive and procrustean sentiment. An appropriate metaphor for the course of nature seems to be less the headlong drive towards an endpoint than a more nuanced interplay between agonistic tendencies. Here the Hegelian dialectic or the Eastern dance between Yin and Yang spring to mind.

Now cognizant of the duality in nature, it no longer becomes necessary to view the production of entropy as a hell-bent rush into nothingness. Increasing entropy also displays a distinctly obverse behavior. Whenever $\alpha$ of a complex dissipative system plots between 1/e and 1, the conventional second law pull toward disorganization will prevail. By strict contrast, if the degree of order in a system falls between 0 and 1/e and it has access to additional energy and resources, increasing entropy will result in augmenting system organization. If the chosen data on ecosystems are sufficiently representative of other natural systems, then available energy and resources give preferential rise to irreversible systems that possess a propitious balance between order and disorganization. Moreover, that favored balance [according to eqn (9)] appears to endow systems with the greatest potential for evolutionary change.

\section{ORGANIZED END STATES}

Of course, energy and resources are not abundant everywhere. Circumstances often arise when one or both are in decline. What then? Classical thermodynamics posits one and only one end - that of complete dissipation or heat death $(\alpha=0)$. The larger picture, however, admits of two possible endpoints - classical heat death or a configuration of perpetual harmony $(\alpha=1)$. In reality, the situation is possibly more complex still. As a system begins to disintegrate, some fragmentary subsystems could decay (classically) into total dissolution, whereas, if the decline in resources is gradual enough, others might settle into enduring equilibrial harmonies.

If the Big Bang narrative by physicists is to be believed, then this last scenario is roughly what led to the formation of matter: The universe began as a chaotic, incredibly dense mass of extremely highenergy photons - pure flux [10]. As this continuum began to expand, some of the photons came together (collided) to form pairs of closed-looped circulations of energy called hadrons - the initial matter and antimatter. Due to a very slight asymmetry that favored matter over antimatter, along with the cooling of the continued expansion, matter came to dominate over antimatter. Continued expansion gave rise to yet larger configurations of matter, all characterized by stable configurations of closed-loop orbitals that could persist indefinitely. The various inequities and asymmetries that dissipated during the course of this evolution are still perceptible in the universal $3 \mathrm{~K}$ background radiation.

\section{THE CRITICALITY OF IRREVERSIBLE FORMS}

Figure 5 is impressive in the way it depicts how narrow is the range of complexity within which living systems thrive. It had earlier been thought that the development of an ecosystem would traverse a wide range of states, culminating in a climax community characterized by a relatively higher value of $\alpha$ [17]. Rather, it now appears that the various 'successional' stages of any ecosystem maintain a narrow overall balance of organization/disorganization. It is possible that such bunching is indicative of Kauffman's [38] suggestion that all living systems are poised near criticality. Should a system 
acquire new structures that would drive it into the supercritical stage, those gains would soon be erased as the system suffers a series of 'avalanches' that drop it back to the near-critical range. Conversely, if a system should fall significantly below criticality, either autocatalytic responses would restore it to near criticality (healing) or it would collapse fully towards $\alpha=0$ (death.)

\section{THE PROSPEROUS WAY DOWN}

The scenario of change during declining availability of resources discussed above is relevant to the current era of declining world oil reserves. Society is being challenged both with diminishing resources as well as with vanishing sinks to receive the products of civilization. There is growing awareness that humanity cannot continue with business as usual. Current attitudes and practices are manifestly unsustainable. How, then, to turn the activities of society in the direction of a more sustainable configuration? The chief problem with many suggestions is that they remain couched within the mindset that created the problem in the first place. For example, some see the way out of society's predicament through the search for increasingly efficient ways of doing things. Such direction makes perfect sense in a monist universe, but the benefits of efficiency become equivocal within a dualist reality. For instance, the degree of order $\alpha$, is a surrogate for overall efficient system performance. Raising $\alpha$ to supercritical levels, however, will only induce avalanche catastrophes that will restore the system to its narrow range of sustainable operation.

Goerner et al. [39] decry that nowhere is such wrong-headedness more evident than in contemporary economics, where unfettered ('efficient') market operation is proffered as the cure for all ills. As with the rest of nature, economics is also a dualist conversation. Matutinović [40] indicates that market efficiency is predominantly achieved through autocatalysis, which drives both $\alpha$ and total activity to higher levels; by now it should be obvious that there is danger in too much of a good thing. Overdevelopment heightens the risks of avalanches that reset the system closer to its critical point. As an antidote, Lietaer et al. [41] propose that the magnitudes and frequency of catastrophic collapses be mitigated by invoking strategies that work against market efficiency, such as alternative currencies.

It should be made clear that no one is contending that efficiency is always undesirable. Rather, as the progression shown in Fig. 3 suggests, it should be sought within the context of declining demands upon the larger environment and diminished overall activity so as to assure what Odum and Odum [42] describe as a 'prosperous way down'.

\section{CHANGING COSMOLOGIES}

Nor is anyone arguing that nature somehow escapes the confines of the second law, which, as Salthe maintains, 'trumps all' [13]. Eddington's warning [1] remains as pertinent as ever - there is indeed a decided bias in the world that favors disorder. What is different under the framework discussed here is that humanity's perspective on the cogent notion of entropy has expanded as ever more complex scenarios are brought under the aegis of a burgeoning science. Within that wider perspective, entropy now behaves in ways that heretofore would have remained overlooked.

Furthermore, the process-based narrative of entropy suggests a new and very different secular eschatology. Over the last century and a half, it has remained a somewhat cynical fashion in many academic circles to celebrate the prospect that the end of the universe story is one of total dissolution and meaninglessness - what John Haught [5] has called 'a cosmology of despair'. To be sure, cosmology is replete with dissipation and chaos - the second law guarantees it! But the wider perspective on that same law reveals an evolving universe wherein new, enduring and meaningful forms can continue to emerge as the universe expands. The universal story thereby grows ever richer as time grades into timelessness and despair is moved over to make room for hope. 


\section{ACKNOWLEDGEMENTS}

The author wishes to thank Stanley Salthe, Daniel Fiscus and an anonymous reviewer for several helpful comments for improving the text. None is responsible for any weaknesses in the theory.

\section{REFERENCES}

[1] Eddington, A.S., The Nature of the Physical World, Macmillan: New York, pp. 74, 1928.

[2] Carnot, S., Reflections on the Motive Power of Heat, ASME: New York, 1824 (translated 1943).

[3] Boltzmann, L., Wissenschaftliche Abhandlungen, J.A. Barth: Leipzig, 1905.

[4] Gibbs, J.W., Elementary Principles in Statistical Mechanics, Ox Bow Press: Woodbridge, Connecticut, 1901 (1981).

[5] Haught, J.F., Science and Religion: In Search of Cosmic Purpose, Georgetown University Press: Washington, DC, 2001.

[6] Schroedinger, E., What is Life?, Cambridge University Press: Cambridge, 1944.

[7] Nicolis, G. \& I. Prigogine, Self-organization in nonequilibrium systems: from dissipative structures to order through fluctuations, Wiley: New York, 1977.

[8] Kauffman, S.A., Investigations, Oxford University Press: Oxford, 2000.

[9] Jørgensen, S.E. \& Mejer, H.F., Exergy as a key function in ecological models. Energy and Ecological Modelling: Developments in Environmental Modelling, eds W.J. Mitsch, R.W. Bosserman \& J.M. Klopatek, Elsevier: Amsterdam, pp. 587-590, 1981.

[10] Chaisson, E.J., Cosmic Evolution: The Rise of Complexity in Nature, Harvard University Press: Cambridge, MA, 2001.

[11] Ulanowicz, R.E., An hypothesis on the development of natural communities. J. Theor. Biol., 85, pp. 223-245, 1980. doi:10.1016/0022-5193(80)90019-3

[12] Fath, B.D., Jørgensen, S.E., Patten, B.C. \& Straškraba, M., Ecosystem growth and development. Biosystems, 77, pp. 213-228, 2004. doi:10.1016/j.biosystems.2004.06.001

[13] Salthe, S.N., Meaning in nature: Placing biosemiotics within pansemiotics. Biosemiotics: Information, Codes and Signs in Living Systems, ed. M. Barbieri, Nova Science Publishers: Hauppauge, New York, pp. 207-217, 2007.

[14] Swenson, R., Emergent attractors and the law of maximum entropy production: Foundations to a theory of general evolution. Systems Research, 6, pp. 187-197, 1989.

[15] Schneider, E.D. \& Sagan, D., Into the Cool: Energy Flow, Thermodynamics, and Life, University of Chicago Press: Chicago, 2005.

[16] Schneider, E.D, \& Kay, J.J., Life as a manifestation of the second law of thermodynamics. Mathematical and Computer Modelling, 19, pp. 25-48, 1994. doi:10.1016/0895-7177(94)90188-0

[17] Ulanowicz, R.E., Ecology, the Ascendent Perspective, Columbia University Press: New York, 1997.

[18] Morowitz, H.J., Energy Flow in Biology, Academic Press: New York, 1968.

[19] Penrose, O., An asymmetric world. Nature, 438, pp. 919, 2005.

[20] Gibbs, J.W., Elementary Principles in Statistical Mechanics, Yale University Press: New Haven, Connecticut, 1902.

[21] Tribus, M., Thermostatics and Thermodynamics, Van Nostrand: Princeton, New Jersey, 1961.

[22] Bateson, G., Steps to an Ecology of Mind, Ballantine Books: New York, 1972.

[23] Ulanowicz, R.E., Goerner, S.J., Lietaer, B. \& Gomez, R. Quantifying sustainability: resilience, efficiency and the return of information theory. Ecological Complexity, 6, pp. 27-36, 2009. doi:10.1016/j.ecocom.2008.10.005 
[24] Shannon, C.E., A mathematical theory of communication. Bell System Tech. J., 27, pp. 379-423, 1948.

[25] Ulanowicz, R.E. \& Norden, J.S., Symmetrical overhead in flow networks. Int. J. Syst. Sci., 1, pp. 429-437, 1990 .

[26] Finn, J.T., Measures of ecosystem structure and function derived from analysis of flows. J. Theor. Biol., 56(2), pp. 363-380, 1976. doi:10.1016/S0022-5193(76)80080-X

[27] Mickulecky, D.C., Network thermodynamics In biology and ecology: an introduction. Ecosystem Theory for Biological Oceanography, eds R.E. Ulanowicz \& T. Platt, Canadian Bulletin of Fisheries and Aquatic Sciences: Ottawa, Vol. 213, pp. 163-175, 1985.

[28] Peirce, C.S., The doctrine of necessity examined. The Monist, 2, pp. 321-338, 1892.

[29] Whitehead, A.N., Modes of thought, The Free Press: New York, 1938 (Paperback 1968).

[30] Popper, K.R., A World of Propensities, Thoemmes: Bristol, 1990.

[31] Kolmogorov, A.N., The local structure of turbulence in incompressible viscous fluid for very large Reynold's numbers. Comptes rendus (Doklady) de l'Académie des Sciences de l'U.R.S.S., 30, pp. 301-305, 1941.

[32] Onsager, L., Reciprocal relations in irreversible processes. Physical Review A, 37, pp. 405-426, 1931. doi:10.1103/PhysRev.37.405

[33] Kauffman, S., At Home in the Universe: The Search for the Laws of Self-Organization and Complexity, Oxford University Press: New York, 1995.

[34] Ulanowicz, R.E., Growth \& Development: Ecosystems Phenomenology, Springer-Verlag: New York, 1986.

[35] Zorach, A.C. \& Ulanowicz, R.E., Quantifying the complexity of flow networks: How many roles are there? Complexity, 8(3), pp. 68-76, 2003. doi:10.1002/cplx.10075

[36] Ulanowicz, R.E., Limitations on the connectivity of ecosystem flow networks. Biological Models, eds A. Rinaldo \& A. Marani, Istituto Veneto de Scienze, Lettere ed Arti: Venice, pp. 125-143, 1997.

[37] Ulanowicz, R.E., Beyond the material and the mechanical: Occam's razor is a double-edged blade. Zygon, 30(2), pp. 249-266, 1995. doi:10.1111/j.1467-9744.1995.tb00068.x

[38] Kauffman, S.A., The Origins of Order: Self-organization and Selection in Evolution, Oxford University Press: New York, 1993.

[39] Goerner, S.J., Lietaer, B., Ulanowicz, R.E. \& Gomez, R., Quantifying economic sustainability: implications for free-enterprise theory, policy and practice. Ecological Economics, in review.

[40] Matutinović, I., The microeconomic foundations of business cycles: from institutions to autocatalytic networks. Journal of Economic Issues, 39(4), pp. 867-898, 2005.

[41] Lietaer, B., Ulanowicz, R.E. \& Goerner, S.J., Options for managing systemic bank crises, http://www.lietaer.com/images/White_Paper_on_Systemic_Bank_Crises_December.pdf, 2008.

[42] Odum, H.T. \& Odum, E.C., A Prosperous Way Down: Principles and Policies, University of Colorado Press: Boulder, Colorado, 2001. 